\title{
Influence of Nanosize Silica Particles on the Rheological Behaviour of Lyotropic Cubic Phase of Glucopone (APG) Surfactant
}

\author{
Mohamed A. Siddig ${ }^{1,2}$, Abubaker A. Siddig ${ }^{3}$, Lal S. Jan ${ }^{4,5}$, Abdelrahman A. Elbadawi ${ }^{1}$, \\ Shahidan Radiman ${ }^{6}$ \\ ${ }^{1}$ Department of Physics, Faculty of Science and Technology, Alneelain University, Khartoum, Sudan \\ ${ }^{2}$ Department of Medical Physics, National University, Khartoum, Sudan \\ ${ }^{3}$ Department of Physics and Astronomy, King Saud University, Riyadh, Saudi Arabia \\ ${ }^{4}$ Govt postgraduate college Mardan, NWFP, Pakistan \\ ${ }^{5}$ Department of general studies, Yanbu Technical Institute, Yanbu Alsinaiyah, Saudi Arabia \\ ${ }^{6}$ Faculty of Science and Technology, National University of Malaysia (UKM), Bangi, Selangor, Malaysia
}

Email address:

Siddig_ma@yahoo.com (M. A. Siddig)

\section{To cite this article:}

Mohamed A. Siddig, Abubaker A. Siddig, Lal S. Jan, Abdelrahman A. Elbadawi, Shahidan Radiman. Influence of Nanosize Silica Particles on the Rheological Behaviour of Lyotropic Cubic Phase of Glucopone (APG) Surfactant. International Journal of Materials Science and Applications. Vol. 4, No. 2, 2015, pp. 130-137. doi: 10.11648/j.ijmsa.20150402.20

\begin{abstract}
The effect of nanosize silica particle on the stability, structures and rheological behaviours of cubic liquid crystalline phases of Glucopone/water/heptane system were investigated. Small-angle X-ray scattering (SAXS) and polarizing microscope were used for phase identification and structure characterization. SAXS scattering profile was characteristic to cubic phase before and after the addition of silica particles. The cubic samples showed several diffraction peaks and can be assigned to the Ia3d space group. The area per surfactant molecule was found to increase as the silica concentrations was increased. Moreover, the rheological study on the cubic phase with different silica concentration was performed. The frequency-dependent storage and loss modulus were found to be characteristic of the cubic phase in the linear viscoelastic region. The cubic samples behave as solid-like material with storage modulus $G^{\prime} \sim 0.7 \times 106$ Pa typical of cubic lyotropic materials. The elasticity of cubic samples was found to increase with silica concentration. The increase in the elasticity was ascribed to the network and the formation of smaller structure as indicated by SAXS measurements. As temperature was further increased a decreased in the elasticity was observed confirming the structural change as indicated by the change in slopes of G' and G'. The cubic samples exhibited shear thinning behaviours, with the dynamic viscosity being affected by the silica particles concentrations and the effect of silica particles on the rheological behaviours was quite noticeable as the concentration increased. The results showed strong correlation between rheology and microstructural changes in the dispersion of nanoparticle silica in the cubic phase system.
\end{abstract}

Keywords: Glycolipid, Rheology, Silica Particles, Small Angle X-Ray Scattering, Viscoelasticity

\section{Introduction}

Glycolipids are ubiquitous membrane constituents of animals and plants that have attracted broad interest in the last few decades $[1,2]$. However, there is a great deal of experimental and theoretical evidence showing that bicontinuous cubic phases may play an important role in processes such as membrane fusion and membrane traffic [3, 4].
The structural investigation of natural glycolipids and the study of their physical properties as well, is a complex matter, providing exciting challenges for biophysicists and biochemists [5].

In recent years, dispersion of silica nano-spheres in liquid crystals has attracted considerable attention [6-8], since this enables the possibility of studying the induced random 
disorder within an ordered phase in a controllable procedure by changing the concentration of silica nano-spheres $[9,10]$.

The effect of various electrolytes and polyols on nonionic hexagonal phase has been previously investigated using small angle X-ray scattering (SAXS) [11]. It is of interest to explore the interaction between colloidal particles and liquid crystalline phases because of the coexistence of colloidal particles with concentrated surfactants in many industrial preparations such as cosmetics,.

The changes in the liquid crystalline properties caused by the embedded silica network have been studied by X-ray scattering [12], optical anisotropy [13], and dielectric measurements [14]. Aerosil, the registered name of Degussa was used in almost all the experiments.

In this work, the cubic phase of the Glucopone/heptane/water ternary system is considered. The phase diagrams of the Glucopone/hydrocarbon/water systems are reported by Siddig et al. [15]. The aim of this study is to investigate the stability, structure and rheology of lyotropic cubic phase of Glucopone nonionic surfactant as a function of silica particle concentration. Optical microscopy and small-angle X-ray scattering (SAXS) were used for phase identification and structure characterization. SAXS experiments are useful in conveying information on the lattice parameter, radius and area per surfactant head group in the cubic liquid crystalline phases. Rheological measurements were performed in order to study the viscoelastic properties of the cubic liquid crystalline phase. In addition, the effect of temperature on the rheological properties of the cubic system was investigated.

\section{Experimental}

\subsection{Material and Samples Preparation}

In this study, the silica particle Aerosil powder (type 380) by Degussa (Germany) was used. Aerosil 380 consists of small silica spheres with a specific surface of $380 \mathrm{~m}^{2} / \mathrm{g}$, average primary particle size $7 \mathrm{~nm}$ and $\mathrm{pH}$ value $3.6-4.3$ (in $4 \%$ water dispersion). Heptane was purchased from Ajax Chemicals (Australia), while technical grade alkyl polyglucosides, APG (commercially known as Glucopone 215 CSUP), was purchased from Fluka. The APG represents a mixture of $\alpha$ - and $\beta$-glucosides [16] and is supplied as 65 wt. $\%$ solution in water. First, the cubic phase of Glucopone/heptane/water was prepared by weighing the surfactant into a glass tube and diluting it to the desired concentration with water or heptane, respectively, by taking into account that the APG is a $65 \mathrm{wt} . \%$ solution in water. Then the silica particles were added as dry phase to the preferred concentrations. The well-mixed samples were then centrifuged in order to remove the bubbles. The samples were heated, thoroughly mixed and centrifuged in wellsealed glass tubes. We report here results from six representative samples with different silica particle concentrations of $0,0.2,0.4,0.8,1.0$ and $2.0 \mathrm{wt} . \%$ as shown in table 1 . All chemical and solvents were of analytical purity and were used as received without further purification.

\subsection{Small Angle X-Ray Scattering}

The liquid crystalline phases were further investigated by SAXS technique at $25{ }^{\circ} \mathrm{C}$ using a Kratky compact smallangle system, which were equipped with a position-sensitive detector containing 1024 channels of width $53.0 \mathrm{~mm}$. The wavelength was $0.154 \mathrm{~nm}$ and the sample-to-detector distance was set at $274 \mathrm{~mm}$.

\subsection{Rheological Measurements}

Rheological measurements in current study were performed with Paar MCR 300 (Physica) apparatus. A coneplate sensor was used with an angle of $1.98^{\circ}$. The sample thickness in the middle of the sensor was $0.049 \mathrm{~mm}$. Oscillation measurements were carried out to give information about storage moduli $G^{\prime}(\omega)$, loss moduli $G^{\prime \prime}(\omega)$, and complex viscosity $\eta^{*}$ in angular frequencies ranging from $0.01 \mathrm{~Hz}$ to $100 \mathrm{~Hz}$.

\section{Results and Discussion}

\subsection{Small Angle X-Ray Scattering}

The Bragg spacing for a cubic lattice with lattice constant $\mathrm{a}_{\mathrm{c}}$, are given by [17]

$$
d_{h k l}=a_{c}\left(h^{2}+k^{2}+l^{2}\right)^{-\frac{1}{2}}
$$

Typical SAXS spectrum is shown in figure 1 for the cubic sample of ternary systems with $0.0 \%$ silica concentration, while Figure 2 shows SAXS spectrum for sample with $1.0 \%$ of nanosize silica concentration. Two peaks are observed and are occurred at $q=0.091$ and $q=0.106$ for the cubic samples with $0.0 \%$ silica of the ternary systems. Combining the SAXS data with the location of the cubic phase in the phase diagram [15], it can remark that the cubic phase is bicontinuous and the hydrophobic/hydrophilic interface has curvature toward oil. A majority of other bicontinuous cubic phase found in other surfactant and lipid systems have been shown to belong to one of these three space groups Ia3d, $\mathrm{Pn} 3 \mathrm{~m}$, and $\mathrm{Im} 3 \mathrm{~m}$ [18]. We attempted an indexing of the observed reflections using the equation for a cubic element cell

$$
\sin ^{2} \theta=\frac{\lambda^{2}}{4 a^{2}\left(h^{2}+k^{2}+l^{2}\right)}
$$

The ratio $\sqrt{ } 6: \sqrt{ } 8$ is matched for the spacing of the sample with $0.0 \%$ silica particles. These values lead to the following indexing (211), (220) and such an indexing would coincide with the space group Ia3d, where the reflections with lower indices are extinguished [19]. The epitaxial relationship between the lamellar phase and the bicontinuous Ia3d is a transformation of (110) plane (lamellar) to the (211) plane in Ia3d [20], i.e. the equivalent lamellar spacing should provide when lattice parameter $a$ divided by $\sqrt{ } 6$. The structural model 
for the bicontinuous cubic phase is required in order to calculate the area per surfactant head group for the cubic phase $[15,17,21]$. The results of the SAXS studies are summarized in Table1.

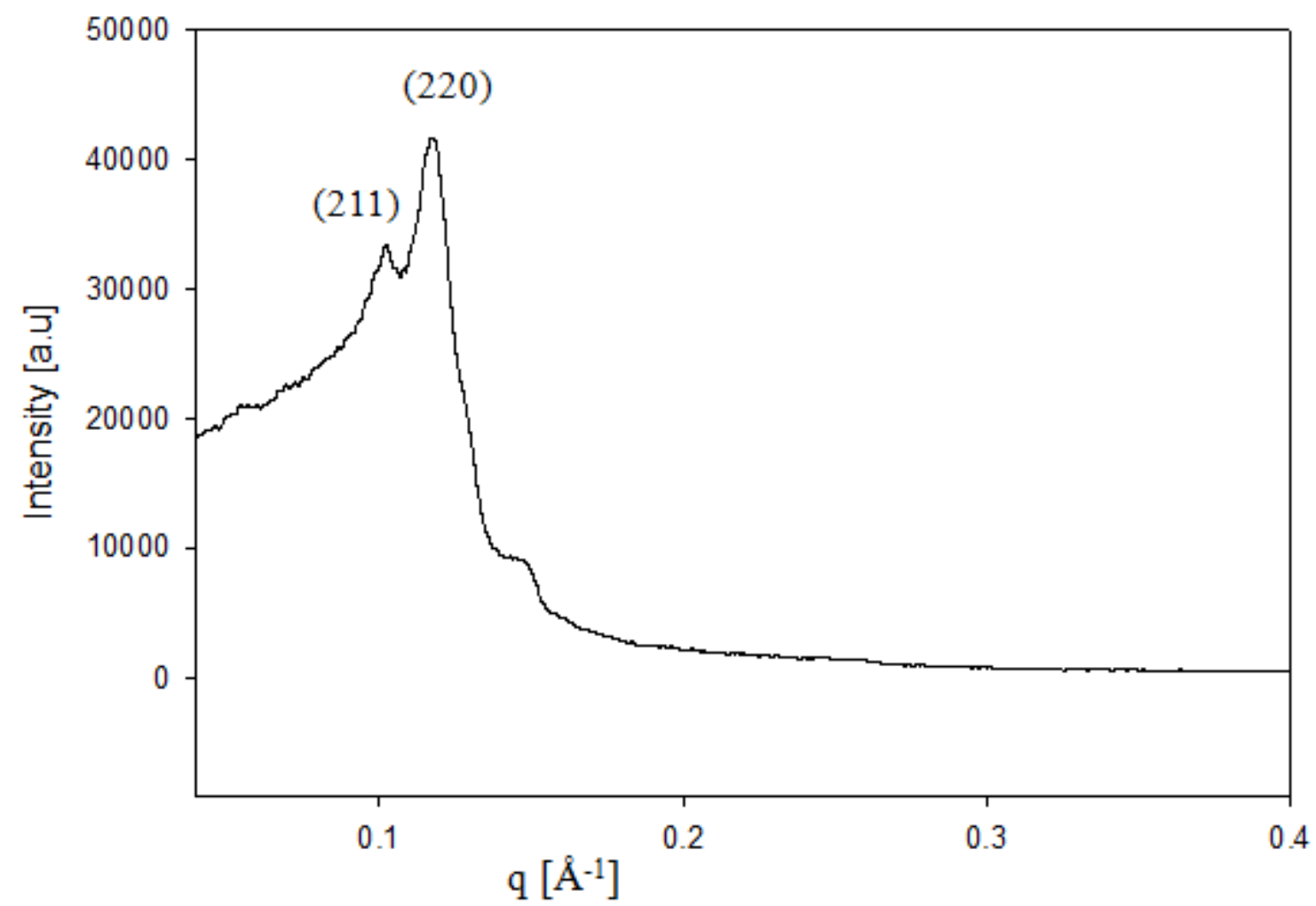

Figure 1. SAXS spectrum for cubic sample with $0.0 \%$ nanosize silica concentration. Two peaks observed and the spacing matches the ratio $\sqrt{6}: \sqrt{ } 8$. These values lead to the following indexing (211), (220).

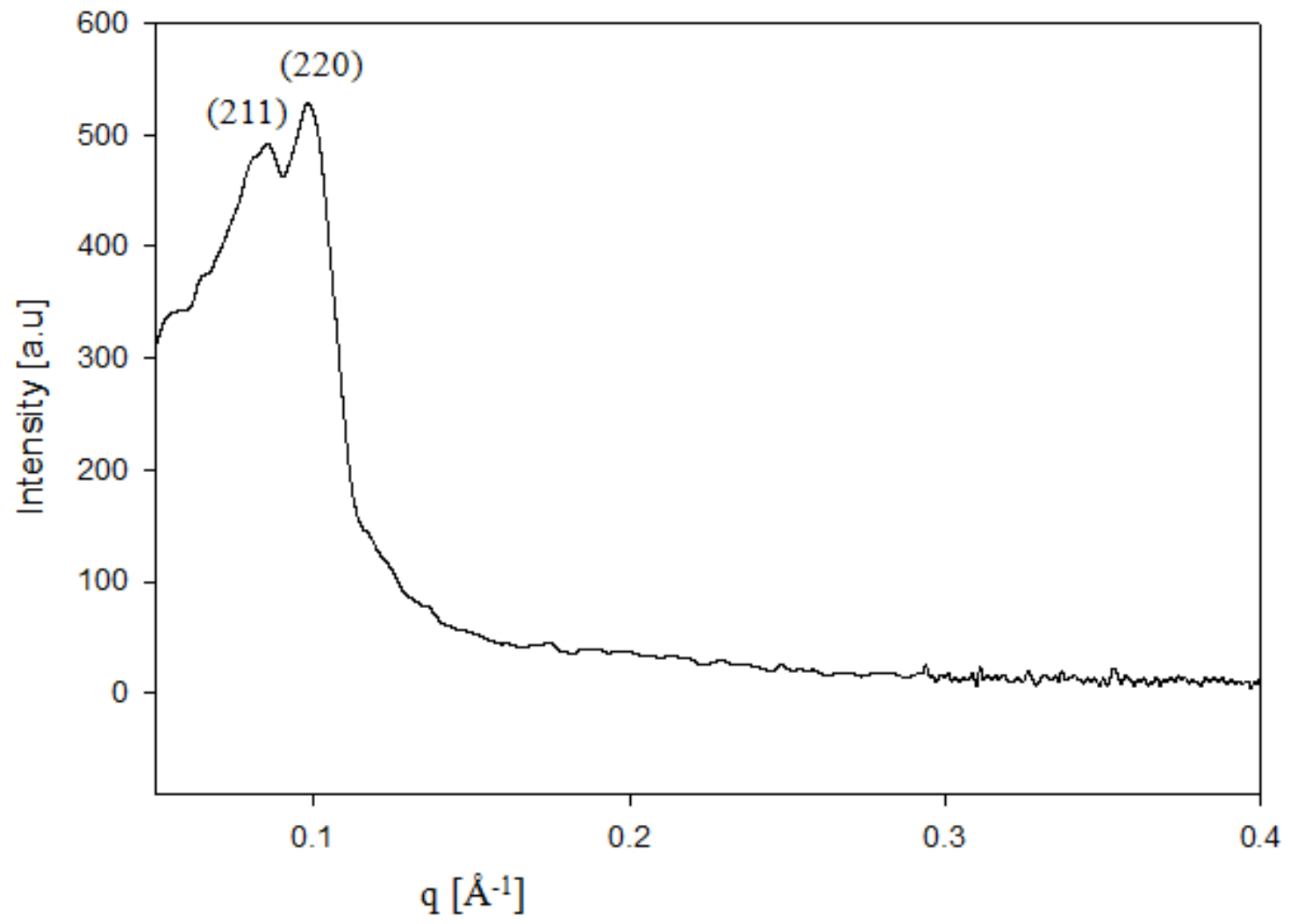

Figure 2. SAXS spectrum for cubic sample with 1.0\% nanosize silica concentration. Two peaks observed and the spacing matches the ratio $\sqrt{6}: \sqrt{ } 8$. These values lead to the following indexing (211), (220). 
Table 1. Summary of the results of SAXS, zero shear viscosity and relaxation time of the cubic phases with different silica concentration.

\begin{tabular}{lllllll}
\hline Sample No. & \multirow{2}{*}{ Silica Concentration } & LC type & Space group & Lattice parameter $\pm \mathbf{0 . 2}(\AA)$ & $\begin{array}{l}\text { Area per head group } \pm \\
\mathbf{0 . 5}\left(\AA^{2}\right)\end{array}$ & $\begin{array}{l}\text { Relaxation time, } \\
\tau / \mathbf{S}\end{array}$ \\
\hline Sample 1 & $0 \%$ & Cubic & Ia3d & 167.6 & 33.3 & 11.1 \\
Sample 2 & $0.2 \%$ & Cubic & Ia3d & 176.7 & 33.3 & 11.1 \\
Sample 3 & $0.4 \%$ & Cubic & Ia3d & 176.7 & 32.5 & 12.5 \\
Sample 4 & $0.8 \%$ & Cubic & Ia3d & 186.9 & 37.4 & 6.6 \\
Sample 5 & $1.0 \%$ & Cubic & Ia3d & 185.4 & 35.7 & 8.3 \\
Sample 6 & $2.0 \%$ & Cubic & Ia3d & 185.2 & 34.9 & 10.0 \\
\hline
\end{tabular}

\subsection{Rheological Properties}

To determine material's linear viscoelastic range, storage and loss modulus are measured as a function of stress. Typical results of the storage and loss modulus as a function of stress at frequency, $\omega$ equal to $1 \mathrm{~Hz}$ are shown in Figure 3. The storage and loss modulus are found to fall off sharply for stress above $3 \times 10^{3} \mathrm{~Pa}$ for samples with different silica concentrations, indicating that the samples have linear viscoelastic range (LVER) up to about $3 \times 10^{3} \mathrm{~Pa}$.

Oscillation experiments used to study the material are subjected to a sinusoidal stress. Figure 4 shows the storage modulus $G^{\prime}(\omega)$, and loss modulus $G^{\prime \prime}(\omega)$ of the cubic crystalline phase as a function of frequency. More elastic behaviours are observed in the range of frequency investigated, and the storage modulus is higher by about two orders of magnitude than the loss modulus at the high frequency range. The formation of the cubic phase is indicated by the high value of elastic modulus $\left(\sim 0.7 \times 10^{6} \mathrm{~Pa}\right)$.

The influence of increasing the silica particles concentration on the elastic properties of the cubic phases is investigated. Six samples of lyotropic cubic phase with different nanosize silica particles concentration are considered (see Table 1). The effect of nanosize silica particles concentrations on the storage moduli for the cubic liquid crystalline phases at $25{ }^{\circ} \mathrm{C}$ is shown figure 5 . It can be observed that all the cubic samples behave as solid-like material with $G^{\prime}$ closed to $0.7 \times 10^{6} \mathrm{~Pa}$, typical of cubic lyotropic materials. The cubic phases behaves as a quasiMaxwellian fluid [22,23]. In this study, similar behavior is observed where $G$ ' reaches plateau at high frequency; $G$ " $>G$, at low frequency even though for some samples it is not obvious where $G^{\prime}$ cross $G$ "' at frequency which is outside the instrument sensitivity frequency range $(<0.01 \mathrm{~Hz})$. With exception of the sample with $2.0 \%$ of silica particles, it can be notice from figure 5 that the elastic modulus is increases with silica particles concentration. This may be due to the formation of smaller structure of cubic phase, which may be is linked to the more solid-like behaviour of the samples. Gradzielski et. al. [24] pointed out that smaller structure lead to higher degree of ordering, which in turn is responsible for the increase in the elastic properties of the system. The elastic modulus is decreased as the concentration of the silica particle is further increased to $2.0 \%$ indicating a more liquidlike behaviour. This effect is obviously associated with the low level of interaction of the oil with the surfactant tails [25]. The frequency dependence of $G$ ' and $G$ " of Maxwell-type fluids given as [26]

$$
\begin{gathered}
G^{\prime}(\omega)=\frac{G_{0} \omega^{2} \tau_{m}^{2}}{1+\omega^{2} \tau_{m}^{2}} \\
G^{\prime \prime}(\omega)=\frac{G_{0} \omega \tau_{m}}{1+\omega^{2} \tau_{m}^{2}}
\end{gathered}
$$

where $G_{0}$ is the 'plateau modulus', $\omega$ the frequency of oscillation and $\tau_{m}$ the relaxation time. A Maxwell fluid is characterized by a single relaxation time. The shape of ColeCole plot, which represent by of plotting $G$ "' against $G$ ', should be a semi circular for ideal Maxwellian model [26]. The distorted circular form (Figure not shown) suggests that the relaxation as characterized in figure 4 is not a single welldefined process, but has some distributions of the relaxation time [27]. In general, from the crossover frequency $\omega_{m}$ (subscript $m$ denotes Maxwell model) the relaxation time $\tau_{m}$ $=1 / \omega$ can be estimated. The relaxation time of the liquid crystalline phases formed by Glucopone systems is shown in Tables 1 . One could probably say that, the relaxation time is decreased with silica concentration.

In order to investigate the effect of temperature on the cubic liquid crystalline phases of the Glucopone ternary systems, the behavior of the storage and loss modulus as a function of frequency for the systems at different temperature is measured. Figure 6 shows the storage and loss modulus as a function of frequency at 25,35 , and $45{ }^{\circ} \mathrm{C}$ for sample with silica concentration of $1.0 \%$. The temperature was increase at a rate of $3{ }^{\circ} \mathrm{C}$ per minute during the measurement. Increasing the temperature results in reduced of the elastic modulus. When the temperature is further increased, the elastic modulus is decrease indicates that the system has more liquid-like behaviours and the crossover occurred at higher frequency which suggests that the relaxation time is decreased.

Complex viscosity, $\eta^{*}$ as a function of frequency for cubic phase with different concentrations of silica particles are plotted in figure 7 . The viscosity shows changes with silica concentration and shear rate as well. Non-Newtonian behavior is observed in all samples, and there is no indication of shear thickening behavior over the entire range of frequency investigated (up to $1000 \mathrm{rad} / \mathrm{S}$ ). Whereas there is significant difference between the viscosities of the samples at low frequency, it appeared from the data that, at high frequency, the viscosity approached a single limiting value. Suspensions of particles in shear flows experience various types of forces such as hydrodynamic forces (including the viscous drag force and particle-particle interaction through 
flow field induced by neighboring particles), colloid chemical forces (including electrostatic, steric and Londonvan der Waals attractive forces), and forces due to gravitational, inertial and thermal or molecular collisional effects [28].

Figure 8 shows the effect of temperature in the complex viscosity as a function of frequency for the sample with 1.0 wt.\% silica. Increasing the temperature from $25^{\circ} \mathrm{C}$ to $35^{\circ} \mathrm{C}$ and further to $45^{\circ} \mathrm{C}$ lead to a normal reduction of the viscosity in the entire frequency range studied. This is may be due to the decrease in the viscosity of the continuous phase and to the thermally induced fluidization of the cubic phase structure.

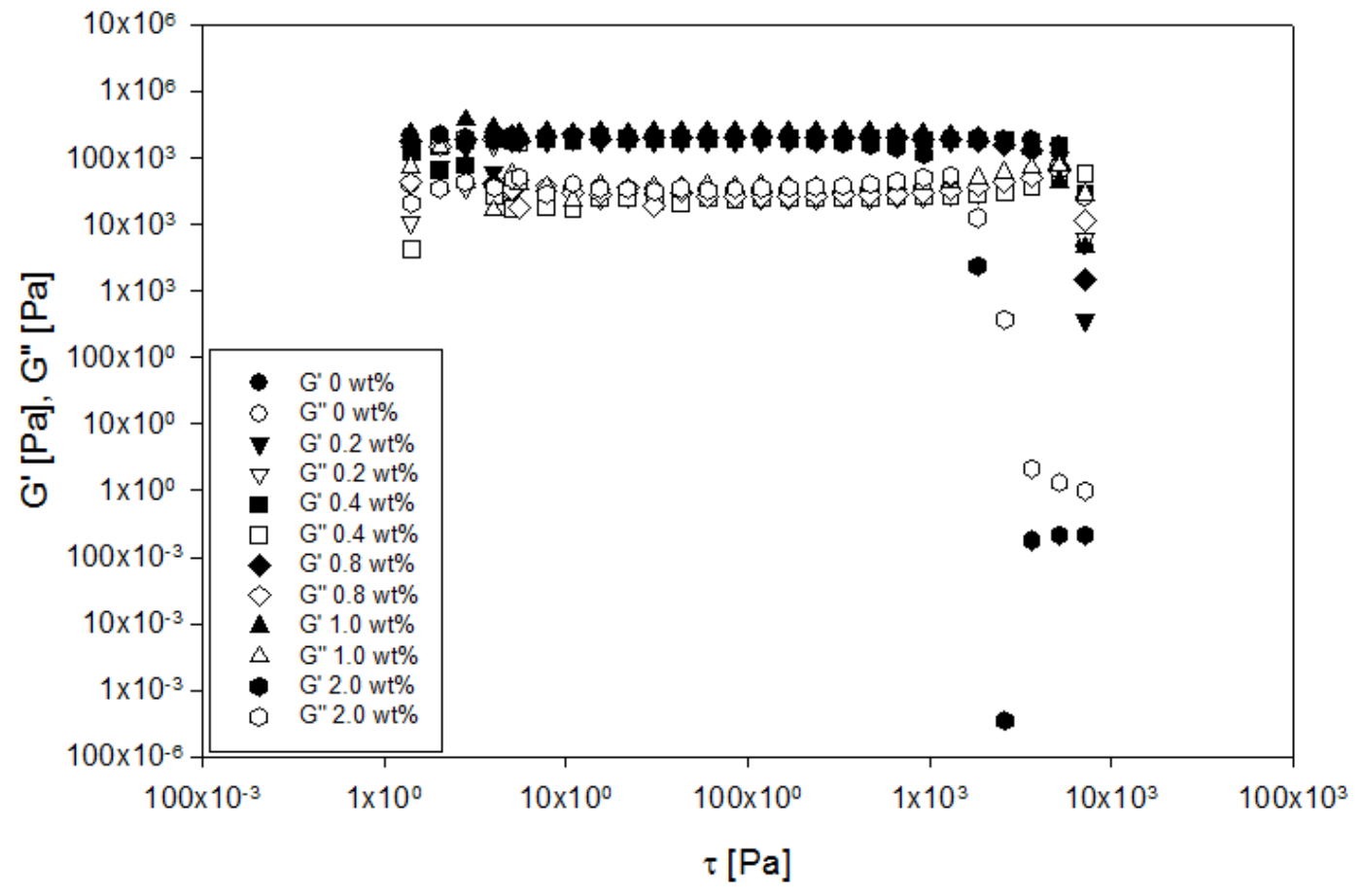

Figure 3. Storage and loss modulus as a function of the applied stress for cubic samples with different silica concentration at $25^{\circ} \mathrm{C}$.

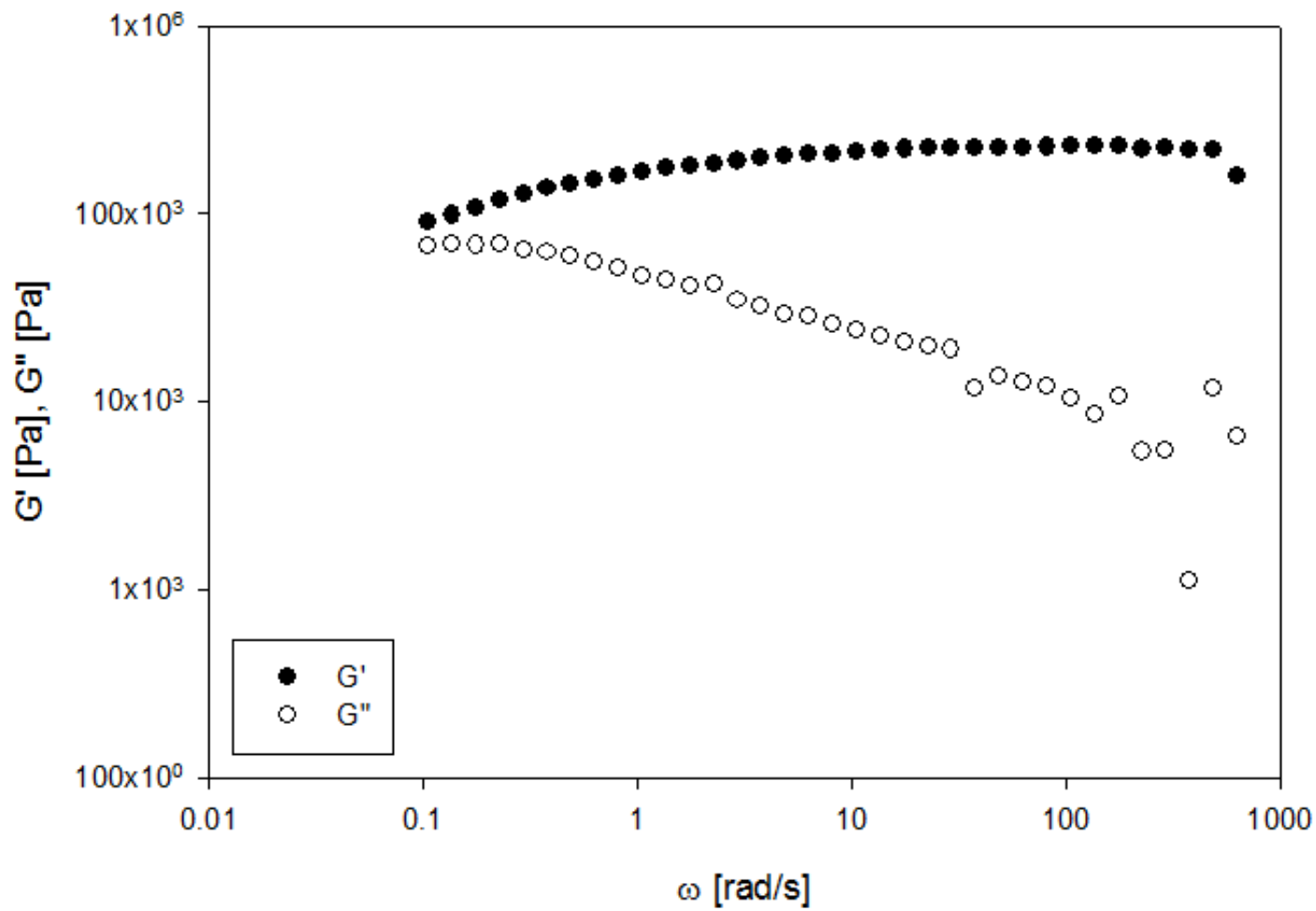

Figure 4. Storage and loss modulus as a function of the applied frequency for cubic sample with $1.0 \%$ silica concentration at $25^{\circ} \mathrm{C}$. 


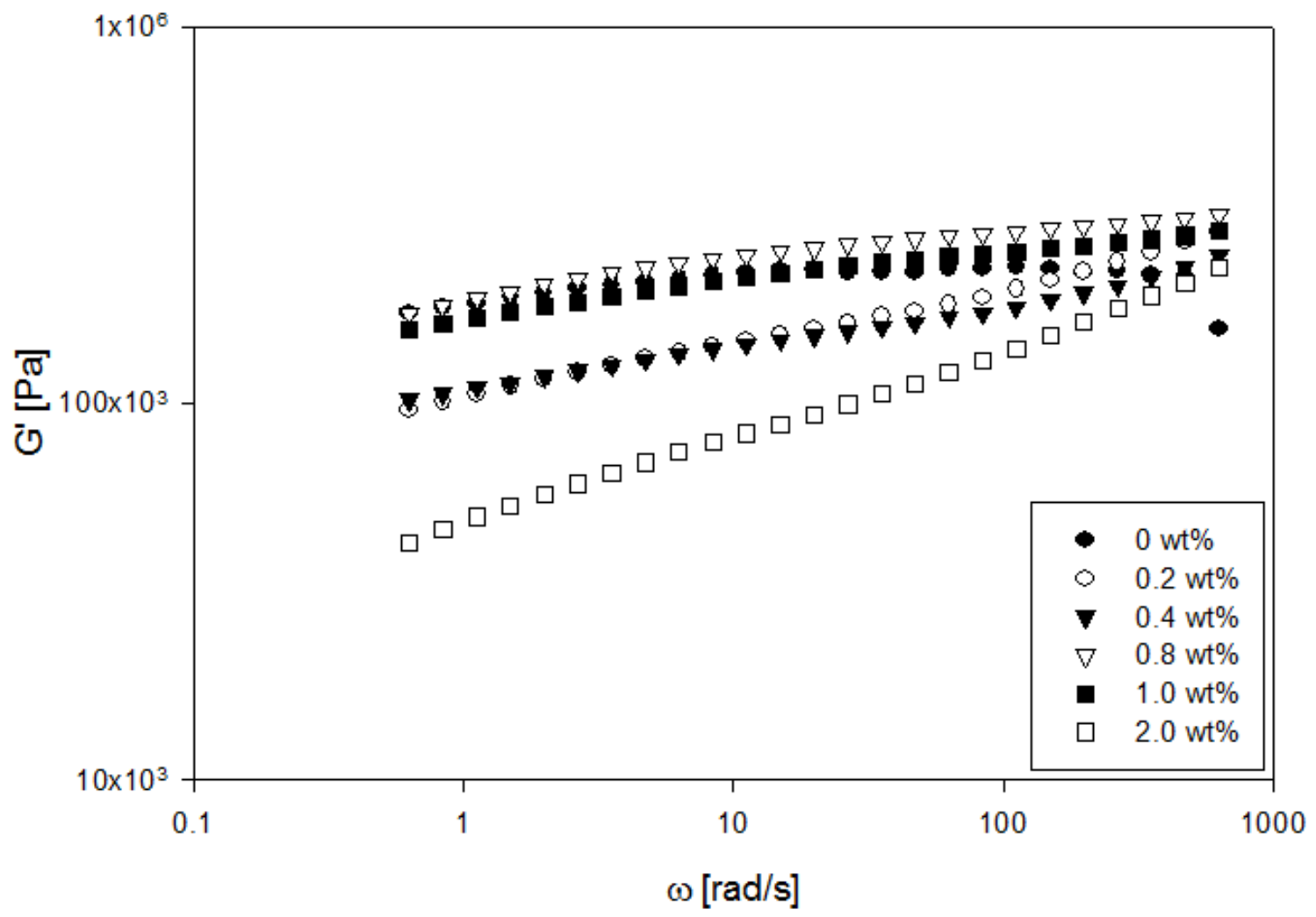

Figure 5. Storage modulus as a function of frequency for cubic samples with different silica concentration at $25^{\circ} \mathrm{C}$.

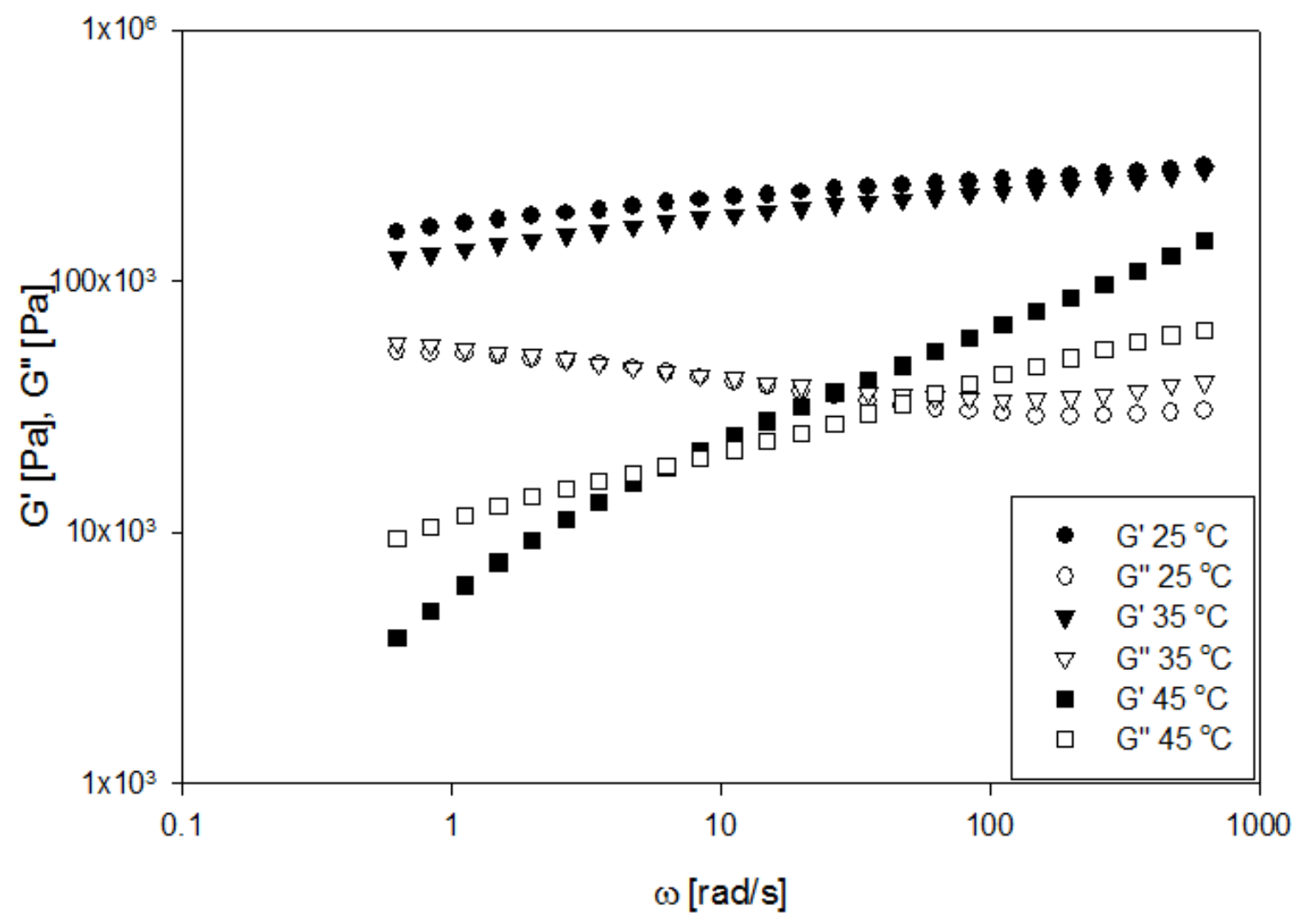

Figure 6. Storage and loss modulus for sample with silica concentration of $1.0 \%$ as a function of frequency for different temperatures. 


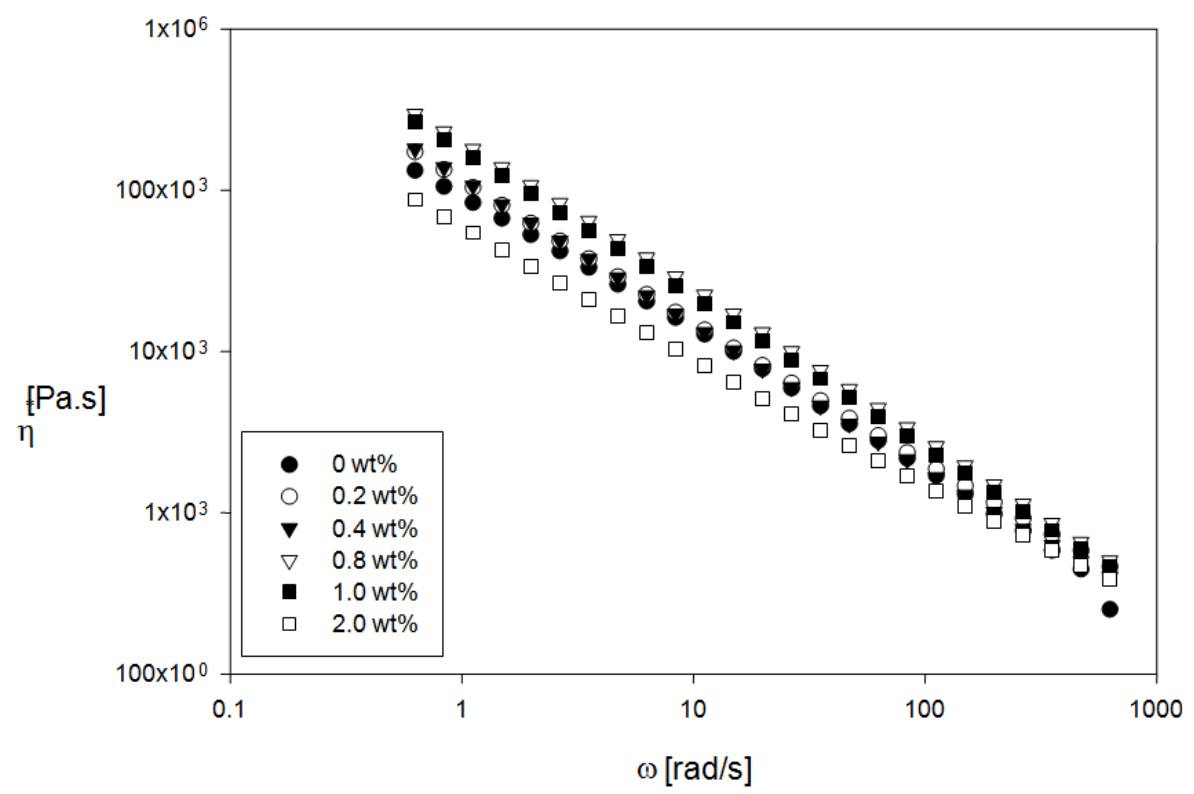

Figure 7. complex viscosities as a function of frequency for cubic sample with different silica concentration at $25^{\circ} \mathrm{C}$.

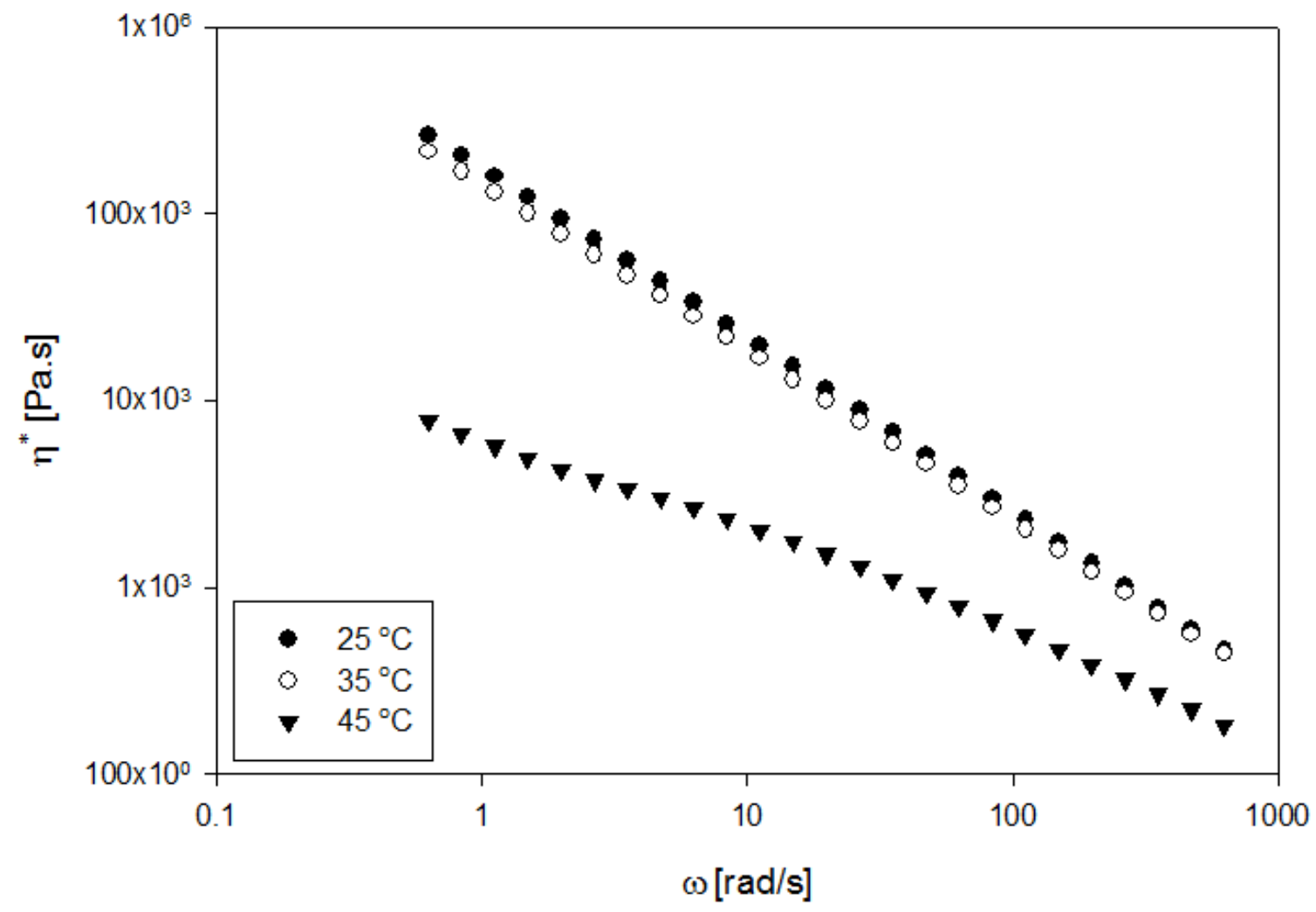

Figure 8. complex viscosities as a function of frequency for cubic sample with 1.0 silica concentration at different temperature.

\section{Conclusion}

The effect of silica particle on the structures of cubic liquid crystalline phases of Glucopone/water/heptane system was studied by means of microscopy, SAXS and rheology. SAXS measurements showed that the area per head groups of cubic phases was found to increase with silica particles concentrations. The frequency-dependent storage and loss modulus were found to be characteristic to the cubic phase in the linear viscoelastic region. The elastic modulus was found to increase with silica concentration which could be linked to strengthening of the networks and the formation of smaller structures. Shear thinning behaviours were observed in the cubic samples and the presence of silica slightly increased the viscosity. Rheological and SAXS scattering data characteristic of cubic phase with different silica particles concentrations were observed to change in similar trends. 


\section{Acknowledgment}

This work was supported by Malaysian Ministry of Science, Technology and Innovation (MOSTI), Malaysia, IRPA 09-02-02-0032-SR0004/04-04.

\section{References}

[1] W. Curatolo, 1987. Biochemica and Biophysica Acta 906: 111136.

[2] W. Curatolo, 1987. Biochemica and Biophysica Acta 906: 137-160.

[3] H. Ellens, D. P.Siegel, D. Alford, P.Yeagle, L. Boni, L. Lis, P. Quinn, \& J. Bentz, 1989. Biochem. 28: 3692-3703.

[4] D. P.Siegel, J. Banschbach, J. Alford, H. Ellens, L. Lis, P. Quinn, P. yeagle, \& J. Bentz, 1989. Biochem. 28: 3703-3709.

[5] C. Tringali, 2001. Biological Compounds from Natural Sources. Isolation, Characterisation and Biological Properties. London: Taylor \& Francis.

[6] J. P.F. Lagerwall, G. Scalia, Current Applied Physics, Volume 12, Issue 6, November 2012, Pages 1387.

[7] F. Muller, J. Degrouard, A. Salonen, Chapter 8: Stabilization of Lipid-Based Lyotropic Liquid Crystalline Phases by Nanoparticles: Perspectives for Colloid-IsasomesIn book: Advances in Planar Lipid Bilayers and Liposomes, Volume 18 , 2013, 209

[8] D. Constantin and P. Davidson, ChemPhysChem 02/2014; 15(7):1270.

[9] T. Iwanaga, M. Suzuki, H. Kunieda, Langmuir, 14 (1998) 5775.

[10] B. R. Midmore, Colloids and Surfaces A: Physicochem. Eng. Aspects, 182 (2001) 83.

[11] E. Anoardo, F. Grinberg, M. Vilfan, R. Kimmich, Chemical Physics, 297 (2004) 99.
[12] G. S. Iannacchione, C.W. Garland, J.T. Mang, T.P. Rieker, Phys. Rev. E, 58 (1998) 5966.

[13] T. Bellini, M. Buscaglia, C. Chiccoli, F. Mantegazza, P. Pasini, C. Zannoni, Phys. Rev. Lett., 85 (2000) 1008.

[14] A. Hourri, P. Jamee, T.K. Bose, J. Thoem, Liq. Cryst., 29 (2002) 459.

[15] M. A. Siddig, S. Radiman, S. V. Muniandy, L. S. Jan, Colloids Surf. A Physicochem. Eng. Aspects, 236 (2004) 57.

[16] A. Stradner, O. Glatter, P. Schurtenberger, Langmuir, 16 (2000) 5354.

[17] F. Nilsson, O. Soderman, I. Johansson, J. Colloid Interface Sci., 203 (1998) 131.

[18] J.M. Seddon, Biochemistry 29 (1990) 7997.

[19] Z. Huang, J.M. Seddon, R.H. Templer, Chem. Phys. Lipids 82 (1996)

[20] J. Charvolin, J. Phys. Chem. 92 (1988) 2646.

[21] F. Nilsson, O. Soderman, I. Johansson, Langmuir, 14 (1998) 4050 .

[22] S. Radiman, C. Toprakcioglu, T. Mcleish, Langmuir, 10 (1994) 61.

[23] J. Jones, T. McLeish, Langmuir, 15 (1999) 7495.

[24] Gradzielski, M. Hofmann, H., Panitz, J. \& Wokaun, A. 1995. Investigations on L2 Phases and Cubic Phases in the System AOT/1-Octanol/Water. J. Colloid interface Sci. 169:103-118

[25] P.J. Maddaford, C. Toprakcioglu, Langmuir 9 (1993) 2868.

[26] M. E. Cates, J. Candau, J. Phys. Condens. Matter, 2 (1990) 6869.

[27] E. Cappelaere, R. Cressely, Rheol Acta, 39 (2000) 346.

[28] M. A. Siddig, S. Radiman, L.S. Jan, S.V. Muniandy, Colloids and Surfaces A: Physicochem. Eng. Aspects 254 (2005) 215 